\title{
Skin Infections and Travelers
}

\author{
Reza Ranjbar', Moein Amoupour ${ }^{1}$, Payam Behzadi ${ }^{1, ~ * ~}$ \\ ${ }^{1}$ Molecular Biology Research Center, Baqiyatallah University of Medical Sciences, Tehran, Iran
}

*Corresponding Author: Payam Behzadi, Molecular Biology Research Center, Baqiyatallah University of Medical Sciences, Tehran, Iran Email: behzadipayam@yahoo.com

\begin{abstract}
Knowing the type of infections in different geographical regions may help to prevent the distribution of diseases. Vaccines, repellents, mosquito nets and clothes are recommended for preventing the increase of infectious diseases. There are different types of climate and geographical items around the world which determine the kind of skin infections. Travelers' skin diseases are recognized as serious global problems. This problem will be solved through an identification of all the types of microbial agents and vectors which may lead to these infections. Informing travelers and advising are the best ways to prevent the spread of infectious diseases.
\end{abstract}

Keywords: Skin Diseases, Travel Medicine, Microbiological Phenomena

Article History: Received: 11 Mar. 2015; Accepted: 30 Apr. 2015; Online Published: 24 Aug. 2015

Cite this article as: Ranjbar R, Amoupour M, Behzadi P. Skin infections and travelers. Int J Travel Med Glob Health. 2015;3(3):99-102.

\section{Introduction}

The skin as an entire and a wide spread organ, blocks the entry of pathogenic microorganisms into in the human body. The type and amount of the skin micro flora are directly in association with the level of skin moisture, temperature, $\mathrm{PH}$ etc. [1]. Diarrhea, fever and skin diseases rank respectively as the first, second and third most common infectious diseases among travelers [2]. Previously, skin diseases were limited in specific geographical regions, but in recent decades the progression of transport technology has led to an increase in the number of travelers in global levels [3]. There is a diversity of reported skin disorders among travelers including allergic rashes, cutaneous larva migrans (CLM), cutaneous leishmaniasis, dengue, dog bites, infectious insect bite reaction, insect bites, rickettsia spotted fever, scabies, skin abscesses, super facial fungal infection, viral chikungunya $[4,5]$.

Patients with skin infections must be checked through a series of questions involving the region and place of travel, the economic level of the travel, the individual behavior and the use of aboriginal cosmetic materials etc. to have an accurate diagnosis. Moreover, the types of clinical evaluations including direct microscopic observation, biopsy, culture, serological investigations are in association with the category of clinical manifestations [4].

\section{Epidemiology}

Insect bites and stings are responsible for a diversity of skin disorders among American travelers [6, 7].

Another investigation indicates that the skin diseases are the third most frequent disease right after systemic febrile disease and acute diarrhea among developing countries. The type and number of skin diseases vary in patients who have traveled; however, the geographical area is considerably important. According to previous reports, the travelers of South Africa and Caribbean regions are more in risk for skin infections diseases [3].

In an international study, the skin infections were reported as the most common diseases after fever and diarrhea among travelers. The most common risk factors for skin infections contain insect bite, skin abscess, allergic reaction and CLM $[7,8]$.

\section{Bacterial Skin Infections}

Bacterial skin infections are known as the most frequent skin disorders among travelers. The clinical manifestations include: necrotizing cellulitis, abscesses, ecthyma, erysipelas, impetigo. The symptoms of skin disorders appear right after infection in the length of travel [8].

In an investigation impetigo, ecthyma or erysipelas were recognized as the most common bacterial cutaneous infections with the total percentage of $75 \%$. The predominant causative bacterial agents of impetigo are recorded as Staphylococcus aureus and Streptococci. Insects' bites are frequent causes of skin lesions which are categorized as the secondary infections or hypersensitivity reactions [9].

S.aureus and Streptococci including Lancefield groups of A, C, G are recognized as the common bacterial agents of skin and soft tissue infections in aged individuals and patients with diabetes [1].

Antibiotics like penicillinase-resistant penicillins, cephalos-porins, clindamycin, or vancomycin (for penicillin sensitive patients) are recommended for a definite and correct treat-ment of streptococcal and staphylococcal skin infections. The skin wounds must be disinfected with antimicrobial agents in parallel with antibiotic therapy $[7,10$, $11]$.

\section{Hookworm Related CLM (HRCLM)}

The HRLCM is recognized as the most common skin disease among travelers who travel to tropical and subtropical regions. HRCLM is caused by nematode larvae entering into the animals' skin in contaminated places. The symptoms occurrence is identified from several days to several months. Localized itching is a common HRCLM symptom which is reported in up to $100 \%$ of sick individuals. Besides, the creeping dermatitis is a specific and differentiated clinical demonstration symptom for recognizing HRCLM disease [7, 8, 12-15].

Edema and vesiculopustular lesions are two key clinical symptoms of larval stage in patients with HRCLM. The outcomes from different investigations show that contaminated soil is the most important risk factor for the infection $[7,15,16]$. 
The HRCLM symptoms often occur in buttocks, feet and tights and usually disappear in a limited range time of 14 days to 2 months. Therefore the clinical diagnostics, clinical manifestations and travel history all together are invaluable items for determining HRCLM. Albendazole, ivermectin (oral consumption) and hiabendazole (topical use) are reported as effective antibiotics for treating the management of HRCLM [7, 9, 12, 15].

\section{Insect Bites and Stings}

Different types of insects with a wide range of bites and stings are seen worldwide. They belong to a special geographical region with their specific life cycles and hosts. The most involved insects with bites include bedbugs, fleas, midges, mosquitoes, sand flies and tsetse. The strength of the host immune system determines the severity of infections and the appearance of allergic reactions. Furthermore, the biochemical and immunological responses differ from person to person. These factors determine the situation of infectious diseases in the form of transitional or long-term period $[4,6]$.

Most insect bites are not painful excluding tsetse and midge bites. People usually are bitten in rural areas and tourism camps. Any contact with animals during the travel may lead to skin infections due to insect bites. In most cases, the bitten areas of skin are accompanied with a release of histamine and sudden inflammations as the natural responses of the immune system in people with severe allergic reactions. Uncovered body parts in travelers are the best points to be bitten by the insects. Studies show that flying insect bites are identified by their cluster patterns while reptiles' bites are detected throughout their linear forms. The itching rounded trace of insects bite is generally accompanied by a central red dot and a pink color peripheral zone involving an area between 1 and $5 \mathrm{~cm}$ in diameter. A series of evidences such as travel history, clinical symptoms and histological examination in association with patients may lead to qualified diagnostic results for identifying skin infectious diseases. The use of insect repellents, mosquito nets, and wearing suitable clothes are the best choices to prevent being bitten by infected insects. Consumption betamethasone and antihistamine are recommended for a rapid and definite treatment [4].

\section{Tsetse Fly Bite}

The skin as an entire and a wide spread organ, blocks the entry of pathogenic microorganisms into in the human body. The type and amount of the skin micro flora are directly in association with the level of skin moisture, temperature, $\mathrm{pH}$ etc. Diarrhea, fever and skin diseases rank respectively as the first, second and third most common infectious diseases among travelers. Previously, skin diseases were limited in specific geographical regions, but in recent decades the progression of transport technology has led to an increase in the number of travelers in global levels [3]. There is a diversity of reported skin disorders among travelers including allergic rashes, cutaneous larva migrans (CLM), cutaneous leishmaniasis, dengue, dog bite, infectious insect bite reaction, insect bites, rickettsia spotted fever, scabies, skin abscesses, super facial fungal infection, viral chikungunya $[1,2,4]$.

Patients with skin infections must be checked throughout a series of questions involving the region and place of travel, the economic level of the travel, the individual behavior and the use of aboriginal cosmetic materials etc. to have an accurate diagnosis. Moreover, the types of clinical evaluations including direct microscopic observation, biopsy, culture, serological investigations are in association with the category of clinical manifestations [4].

\section{Abscesses}

Individual hygiene and economic situation of travelers determine personal health conditions. Moreover, insect bites and trauma are two significant environmental factors for the appearance of abscesses. In recent years, travelers get more abscesses. Staphylococcus aureus is recognized as a common bacterial agent for traveler's abscesses. The abscesses caused by panton-valentine leukocidin toxin producing strains of S.aureus may be treated by drainage. Antibiotic therapy is suggested for people who are considered the carriers of S.aureus on their skin or within their noses $[4,17]$.

\section{Cutaneous Leishmaniasis}

Leishmania genus is an obligate intracellular protozoon which may lead to leishmaniosis. Although, there are different types of leishmaniosis, cutaneous leishmaniosis is a common skin infection among travelers who travel to tropical and sub-tropical regions such as the Middle East countries, Southern Europe, and Latin America. This infection is transmitted by Sand flies [7, 18-20].

Skin wounds and lesions with or without pain, generally appear in a period of weeks, months or years. Sometimes, satellite lesions are demonstrated around central main wounds which are useful for clinical diagnosing. The wounds are often limited by themselves. A series of clinical demonstrations, diagnostic tools like PCR, culture, and direct microscopy and travel history are needed for accurate diagnosis. Insect repellents, mosquito nets, long sleeves and long pants are necessary for protecting travelers from being infected. Amphotericin B, and miltefosine are appropriate antibiotics for treatment [18-20].

\section{Myiasis}

Myiasis, a tropical and sub-tropical disease occurred by diptera fly larvae is common among animals (vertebrates) and humans. Furuncular myiasis is recognized as travelers' skin infectious disease which is transmitted by Cordylobia anthropophaga larvae and Dermatobia hominis larvae in Africa and Latin America, respectively. Economic status and social behaviors of travelers are significant risk factors for being infected by myiasis. This kind of skin infection has been ranked fifth among travelers' skin diseases around the world. Clinical symptoms of myiasis depending on larvae types are varied from 7 to 21 days. The use of ivermectin and performing surgery are recommended for a definite treatment [7, 21-24].

\section{Tungiasis}

Tungiasis is an infectious disease caused by pregnant female sand fleas of Tunga penetrans (chigoe and jigger fleas). The fleas feed on warm blooded hosts and generally bite their feet. Therefore, walking with naked feet on contaminated soil and sand in tropical and sub-tropical areas such as Africa, Asia, the Caribbean zone, India and Latin America is recognized as a considerable risk factor. The bitten skin involves warty nodules and black centered papules. The clinical symptoms are appeared in a period of 7 
to 40 days. Clinical demonstrations and morphological characteristics of fleas are suitable for the diagnosing tungiasis. A definite treatment is done by extraction of fleas $[7,22]$.

\section{Tick Bites}

Tick is a giant gobbler which feeds on humans' and animals' blood. The people who work with animals are predisposed for being bitten by ticks. Ticks are categorized into soft and hard bodied. Hard ticks are recognized as the most significant vectors for their bites. As the tick bites are not painful, there is no alert for notifying patients about disease transmission. Diseases like the African tick fever, the Rocky Mountain spotted fever, and the Lyme are transmitted by tick bites $[4,6]$.

\section{Lyme Disease}

The Lyme disease as a bacterial skin infection is caused by Borrelia burgdorferi; the bacterial agent is transmitted into the human host by Ixodes bites. In most cases, patients have no idea about the way of infection. Tropical and sub-tropical zones of South Scandinavia, Northern Mediterranean areas and etc. are recognized as an endemic foci for Lyme disease. The infection is reported from small groups of travelers [2527].

Lyme disease clinical demonstrations include myalgia, arthralgia, fever, and headaches with rashes. The progression of the Lyme disease may lead to neurological disorders and cardiac malfunctions [25-27].

The use of insect repellents, long sleeves, long pants, and socks is suggested for not being bitten.

Travel history, clinical manifestations and serological techniques are appropriate approaches for accurate diagnosis. Consumption of doxycycline ceftriaxone is recommended for a definite treatment [25-27].

\section{Mite}

Mite as a small arthropod is fed on different animals and mammals. Chickens, dogs, rats, mice and etc. are the main hosts, while humans are occasional hosts for mites. Burning and itching are two general manifestations of mites' bites. Antihistamines and betamethasone are recommended as proper choices for treatment $[4,6]$.

\section{Scabies}

Human scabies is an infection caused by the Sarcoptes scabiei mite. Scabies demonstrations are accompanied by a severe itching. The female mites may lead to infection in their hosts. The mite eggs and its feces are responsible for significant allergic reactions on the host's skin. Intense itching usually appears at night $[4,28]$.

The scabies symptoms are observed on different parts of the skin particularly burrow wounds on wrist, trunk and genitals areas. The severe or crusted scabies is in association with skin infestation by hundreds of female mites. The clinical manifestation included widespread crusted plaques on trunk area, erythema, and itching. The infection is often observed in aged individuals and people with a defective immune system. Observation of extracted mites within isolated samples in the presence of aforementioned clinical demonstrations confirms the disease of scabies [4, 28].

Permethrin $(5 \%)$ or Malathion $(0.5 \%)$ are suitable choices for treatment. The topical use of aforementioned agents must be done by the family members and they must be exposed from the neck down. For treating crust scabies, the oral consumption of ivermectin is recommended. The use of ivermectin in young children (under 2 years) and pregnant women is forbidden. In parallel with treatment, bed covers and underclothes must be washed. The itchy skin is normally treated in a period of 30-40 days [4, 29].

\section{Skin Disorders in Association with Usual Systemic Febrile Illness}

\subsection{Arboviral Infectious Diseases}

Arboviral infectious diseases have been increased throughout the raise up of dengue and chikungunya viruses' infections among travelers. Arthropods are the most important vectors for the aforementioned viral infectious agents [7].

\subsection{Dengue}

Mosquitoes are well known vectors for quick spread of dengue viruses. Today, the expansion of dengue viruses is seen in rural and urban areas in different geographical regions including Africa, the tropical climates of Asia, Latin America, and the Mediterranean zone [30].

Aedes mosquitoes are recognized vectors of dengue viruses. The dengue fever's main symptoms involve a sudden appearance of fever, rash, headache, and myalgia etc. which are usually revealed within 2-4 days. The detection of infection must be performed in the earliest time. The serological tests are used for the identification of dengue fever [3, 31].

\subsection{Chikungunya}

The Chikungunya virus is the causative agent of a systemic febrile illness with some skin disorders. The virus vector is Aedes mosquitoes. The Chikungunya virus has the same geographical distribution as the dengue virus [32].

There are some similarities between the dengue fever and the chikungunya virus disease including sudden high fever and erythematous rash. Therefore, the clinical manifestations of both infections are close. The particular demonstration relating to the chikungunya infection is polyarthritis which may continue several months with some disability. In few cases, some abnormalities such as heart failure, variable hypertension, acute renal failure, hepatitis, etc. may occur. Complications relating to the infection are more usual in elderly patients. Cell culture, PCR and serological assays are the most common techniques for detecting the Chikungunya virus. The use of nonsteroidal anti-inflammatory and steroid drugs is recommended for treatment $[3,32,33]$.

\subsection{Rickettsiosis and Scrub Typhus}

Rickettsiosis is known as a common zoonotic bacterial infection among travelers. The infection is transmitted to human hosts by arthropod vectors. Rickettsiosis is an urgent disease which is generally accompanied by fever and diffuse rash. In some reports, organ failures and mortality among infected people are recorded. A type of rickettsiosis is occurred due to the African tick bite fever [34, 35].

The disease is caused by the bacterial agent of Rickettsia africae which its vector is the cattle tick of Amblyomma. The infection is endemic in some regions of Africa and the Caribbean. The clinical manifestations include fever, headache, neck myalgia, scars, and occurrence of maculopapular or vesicular rash [7, 34, 36].

\subsection{Different Types of Rickettsiosis in Infected Travelers}

Rickettsia conorii, the Mediterranean spotted fever microbial agent is an endemic disease recognized in the Euro-Mediterranean region, Africa and Asia. This disease is 
transmitted via dog bites [7, 37].

Rickettsia typhi, the bacterial agent of murine typhus disease is transmitted through fleas. The distribution of infection is recognized in climate zones of tropical and subtropical regions [7, 37].

Rickettsia rickettsii is recognized as the bacterial agent of Rocky Mountains which is transmitted by dog bites. The infection is endemic in the American continent [7, 37].

Orientia tsutsugamushi is the microbial agent of Scrub typhus which is transmitted by trombiculid mite larvae bites. The disease is endemic and distributed in the Western Pacific and South Asia. Clinical symptoms and serological assays provide routine diagnostic approaches. However, PCR techniques are rapid diagnostic methods for detecting the microbial agents within scars taken from skin biopsies.

Doxycycline is an appropriate antibiotic for treating adults; however, it is not permitted for children [7, 38].

\section{Conclusion}

Skin infections are a considerable health care problem among global travelers. The most reported data are provided from medical centers, in which doctors visit infected travelers turned back from the trips. In the following of detection of clinical manifestations relating to tropical infectious diseases in patients, some questions including travel history, time of travel, visited regions, probable risk factors, and self-medication must be asked from them. Travelers must be advised for the types of infectious diseases by medical health centers before their trips.

\section{Acknowledgments}

This paper is a part of seminar class of the MSc students of the Medical Biotechnology, Molecular Biology Research Center, Baqiyatallah University of Medical Sciences.

\section{Authors' Contributions}

Moein Amoupour drafted the primary version of the manuscript. Payam Behzadi helped Moein Amoupour to complete the paper and Dr. Reza Ranjbar supervised and finalized the writing of the paper.

\section{Financial Disclosure}

None.

\section{Funding/Support}

Not declared.

\section{References}

1. Dryden MS. Skin and soft tissue infection: microbiology and epidemiology. Int J Antimicrobial Agents. 2009;34:S2-S7.

2. Herbinger KH, Siess C, Nothdurft H, Von Sonnenburg F, Löscher T. Skin disorders among travellers returning from tropical and nontropical countries consulting a travel medicine clinic. Trop Med Int Health. 2011;16(11):1457-64

3. Kovarik C. Cutaneous infectious diseases in the returning international traveler. Cutis. 2011;87(3):112-3.

4. Morris-Jones R, Morris-Jones S. Travel-associated skin disease. Infect Dis Clin North Am. 2012;26(3):675-89.

5. VanRooyen MJ, Venugopal R. World Travelers: Introduction.

6. Burns Bo D, Williams RA. Insect bites 2015 [cited 2015]. Available from: http://emedicine.medscape.com/article/769067-overview.

7. Hochedez P, Caumes E. Common skin infections in travelers. J Travel Med. 2008;15(4):252-62.

8. Freedman DO, Weld LH, Kozarsky PE, Fisk T, Robins R, von Sonnenburg F, et al. Spectrum of disease and relation to place of exposure among ill returned travelers. $\mathrm{N}$ Engl $\mathrm{J}$ Med. 2006;354(2):119-30

9. Keystone JS. Skin and Soft Tissue Infections in Returned Travelers.
CDC Health Information for International Travel 2012: The Yellow Book: The Yellow Book. 2011:463.

10. Stevens DL, Bisno AL, Chambers HF, Everett ED, Dellinger P, Goldstein EJ, et al. Practice guidelines for the diagnosis and management of skin and soft-tissue infections. Clin Infect Dis. 2005;41(10):1373-406.

11. Traveler information. Skin disease in travelers 2012 [cited 2015]. Available from: https://healthservices.appstate.edu/files/filecabinet/folder19/Travele r_Information__Skin_Diseases_in_Travelers.pdf.

12. Hochedez P, Caumes E. Hookworm-Related Cutaneous Larva Migrans. J Travel Med. 2007;14(5):326-33.

13. Bouchaud O, Houzé S, Schiemann R, Durand R, Ralaimazava P, Ruggeri $\mathrm{C}$, et al. Cutaneous larva migrans in travelers: a prospective study, with assessment of therapy with ivermectin. Clin Infect Dis. 2000;31(2):493-8.

14. Green AD, Mason C, Spragg PM. Outbreak of cutaneous larva migrans among British military personnel in Belize. J Travel Med. 2001;8(5):267-9.

15. Tremblay A, MacLean J, Gyorkos T, Macpherson D. Outbreak of cutaneous larva migrans in a group of travellers. Trop Med Int Health. 2000;5(5):330-4

16. Blackwell V, Vega-Lopez F. Cutaneous larva migrans: clinical features and management of 44 cases presenting in the returning traveller. Br J Dermatol. 2001;145(3):434-7.

17. Behzadi P, Behzadi E. Environmental Microbiology. Tehran, Iran: Niktab Press; 2007. Persian

18. Herwaldt BL, Magill AJ. Leishmaniasis, Cutaneous 2015 [updated 2015; cited 2015]. Available from: http://wwwnc.cdc.gov/travel/yellowbook/2016/infectious-diseasesrelated-to-travel/leishmaniasis-cutaneous.

19. Magill AJ. Cutaneous leishmaniasis in the returning traveler. Infect Dis Clin North Am. 2005;19(1):241-66.

20. Blum J, Desjeux P, Schwartz E, Beck B, Hatz C. Treatment of cutaneous leishmaniasis among travellers. J Antimicrob Chemother. 2004;53(2):158-66.

21. Francesconi F, Lupi O. Myiasis. Clin Microbiol Rev. 2012;25(1):79105.

22. Diaz JH. The epidemiology, diagnosis, management, and prevention of ectoparasitic diseases in travelers. J Travel Med. 2006;13(2):100 11

23. Tamir J, Haik J, Schwartz E. Myiasis with Lund's fly (Cordylobia rodhaini) in travelers. J Travel Med. 2003;10(5):293-5.

24. Maier H, Hönigsmann H. Furuncular myiasis caused by Dermatobia hominis, the human botfly. J Am Acad Dermatol. 2004;50(2):26-30.

25. Mead PS. Lyme Disease 2015 [updated 2015; cited 2015]. Available from: http://wwwnc.cdc.gov/travel/yellowbook/2016/infectiousdiseases-related-to-travel/lyme-disease.

26. Karami A, Sarshar M, Ranjbar R, Zanjani RS. The Phylum Spirochaetaceae. The Prokaryotes: Springer; 2014. p. 915-29.

27. Sorouri R, Ramazani A, Karami A, Ranjbar R, Guy EC. Isolation and characterization of Borrelia burgdorferi strains from Ixodes ricinus ticks in the southern England. BioImpacts: BI. 2015;5(2):71.

28. Parasites - Scabies 2010 [cited 2015]. Available from: http://www.cdc.gov/parasites/scabies/.

29. Sharma R, Singal A. Topical permethrin and oral ivermectin in the management of scabies: a prospective, randomized, double blind, controlled study. Indian J Dermatol. 2011;77(5):581.

30. Organization WH, Research SPf, Diseases TiT, Diseases WHODoCoNT, Epidemic WHO, Alert P. Dengue: guidelines for diagnosis, treatment, prevention and control: World Health Organization; 2009.

31. Thiboutot MM, Kannan S, Kawalekar OU, Shedlock DJ, Khan AS, Sarangan G, et al. Chikungunya: a potentially emerging epidemic. PLoS Negl Trop Dis. 2010;4(4):e623.

32. Guha-Sapir D, Schimmer B. Dengue fever: new paradigms for a changing epidemiology. Emerg Themes Epidemiol. 2005;2(1):1.

33. Campion EW, Weaver SC, Lecuit M. Chikungunya virus and the global spread of a mosquito-borne disease. N Engl J Med. 2015;372(13):1231-9.

34. Ericsson CD, Jensenius M, Fournier P-E, Raoult D. Rickettsioses and the international traveler. Clin Infect Dis. 2004;39(10):1493-9.

35. Fournier P-E, Roux V, Caumes E, Donzel M, Raoult D. Outbreak of Rickettsia africae infections in participants of an adventure race in South Africa. Clin Infect Dis. 1998;27(2):316-23.

36. Jensenius M, Fournier P-E, Vene S, Hoel T, Hasle G, Henriksen AZ, et al. African tick bite fever in travelers to rural sub-Equatorial Africa. Clin Infect Dis. 2003;36(11):1411-7.

37. Dobler G, Wölfel R. Typhus and other rickettsioses. Dtsch Arztebl Int. 2009;106(20):28

38. Typhus S. Diagnosis and treatment of scrub typhus-the Indian scenario. J Assoc Physicians India. 2010;58:11. 